\title{
Implantación de un programa de prostatectomía radical laparoscópica
}

\author{
Cansino Alcaide JR, Cabrera Castillo PM, Álvarez Maestro M, Martínez-Piñeiro Lorenzo L, \\ Cisneros Ledo J, De la Peña Barthel J.
}

Servicio de Urología. Hospital Universitario La Paz. Madrid

Actas Urol Esp 2006; 30 (5): 469-473

\section{RESUMEN}

IMPLANTACIÓN DE UN PROGRAMA DE PROSTATECTOMÍA RADICAL LAPAROSCÓPICA

En el deseo de éste artículo, está expresar nuestra experiencia a la hora de poner en práctica un programa de prostatectomía radical laparoscópica. Pensamos que puede ser interesante, de cara a implantar un programa de éste tipo, conocer cuáles fueron nuestras dificultades al inicio.

Esperamos que en la medida de lo posible, podamos facilitar ésta difícil tarea, explicando qué pasos hemos seguido y cuáles son nuestras conclusiones y recomendaciones

Palabras clave: Laparoscopia. Cáncer de próstata. Prostatectomía radical

\begin{abstract}
DEVELOP OF A LAPAROSCOPIC RADICAL PROSTATECTOMY PROGRAM IN AN UNIVERSITY CENTER

The aim of this article is to report our experience in setting up a laparoscopic radical prostatectomy programme. We believe that knowledge of the difficulties we faced at the start will be useful for those who wish to implement a programme like ours. We hope that by explaining the steps we took as well as our conclusions and recommendations this difficult task can be made easier.
\end{abstract}

Keywords: Laparoscopy. Prostate cancer. Radical prostatectomy.

$\mathrm{L}$ a prostatectomía radical laparoscópica se encuentra dentro de los procedimientos laparoscópicos más difíciles en la urología. Antes de iniciar un programa de prostatectomía radical laparoscópica en humanos se debe realizar un entrenamiento adecuado de los cirujanos responsables

\section{PASOS SEGUIDOS PARA IMPLANTAR EL PROGRAMA DE PROSTATECTOMÍA RADICAL LAPAROSCÓPICA EN EL HOSPITAL LA PAZ}

En el Servicio de Urología del Hospital Universitario $\mathrm{La} \mathrm{Paz}$, se realizan aproximadamente 100-150 prostatectomías radicales al año y se prevé que la cifra no disminuya en el futuro, incluso que aumente, dado que cada vez realizamos el diagnóstico más precozmente.
El programa de prostatectomía radical laparoscópica, se inició en nuestro servicio en junio de 2002, llevando realizados casi 600 casos en febrero de 2006. Desde el principio nos planteamos finalizar todas las cirugias sin intentar la reconversión planificada.

Casi todas las cirugias han sido realizadas de forma transperitoneal, siendo los casos realizados mediante la técnica descendente (similar a la realizada en Montsouris y Bourdeos posteriormente) y con técnica mixta retrógrada-anterógrada (Heilbronn modificada).

Se designaron al inicio del programa a dos urólogos del servicio, con el fin de no paralizar el programa en ausencia de alguno de ellos.

Ambos debieron realizar un entrenamiento intensivo en simulador de laparoscopia, en animales de experimentación, así como sendas rota- 
ciones en centros con experiencia en prostatectomías radicales laparoscópicas durante 1 mes, antes de iniciar nuestro programa en humanos.

El tiempo total de entrenamiento fue de 6 meses.

El entrenamiento en simulador constó, para cada uno de los cirujanos, de unas 200 horas, en las que se trabajó fundamentalmente la anastomosis cérvico-uretral usando como modelo los pabellones de dos sondas vesicales cortadas. Una vez se consiguió destreza para la anastomosis en el simulador (de 7 a 9 puntos en menos de 45 minutos) se realizó una primera tanda de cirugía en 5 animales de experimentación. (Figs. 1 y 2).

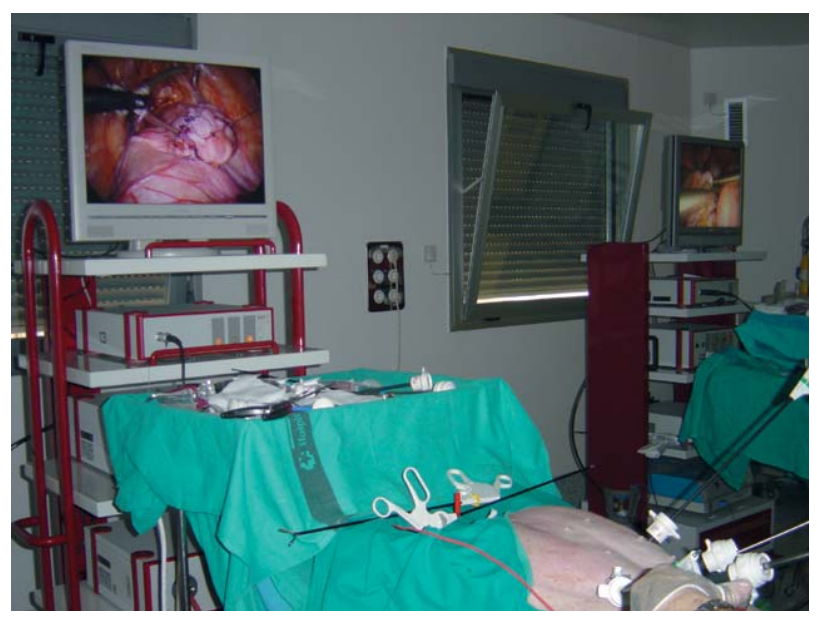

FIGURA 1: Una vez practicado en pelvitrainer, se realizarán en cerdas, modelos de anastomosis vesicouretral.

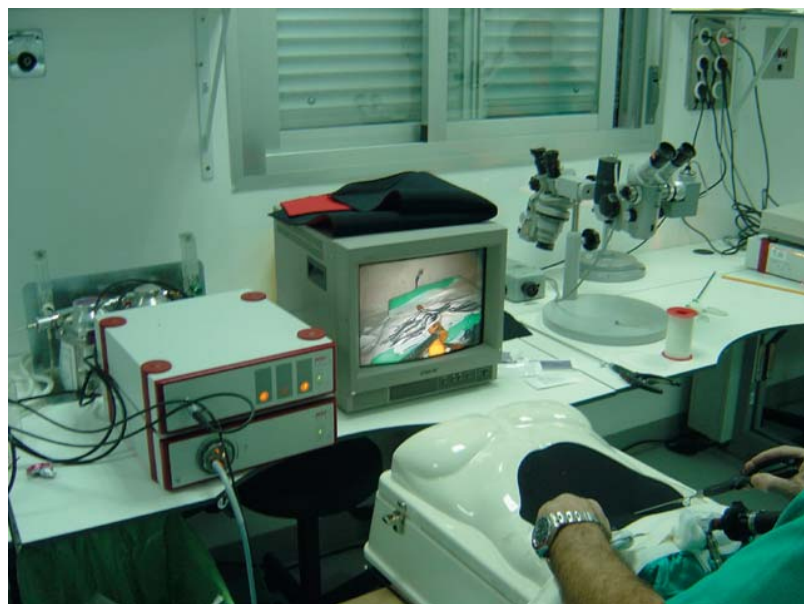

FIGURA 2: No se debe abandonar la práctica de suturas, a pesar de tener cierta experiencia, ya que se llega cansado a ésta parte y frecuentemente es motivo de reconversión a cirugía abierta.
Dado que la próstata en el cerdo no es un buen modelo para la disección utilizamos 2 modelos de experimentación distintos en hembras (Figs. 3, 4, 5 y 6). Por un lado, la anastomosis cérvico-uretral ( 2 anastomosis por cada animal); por otro lado, la sutura de las dos trompas uterinas a vejiga realizando una anastomosis cada uno de los cirujanos y cambiando el papel de ayudante y cirujano. En cada animal se realizaban por lo tanto 4 anastomosis (Figs. 6, 7 y 8).

Con la experiencia obtenida los 2 urólogos designados rotaron durante aproximadamente 1 mes en sendos centros con experiencia en prostatectomía radical laparoscópica. Una vez contrastadas las experiencias de ambos, se completó

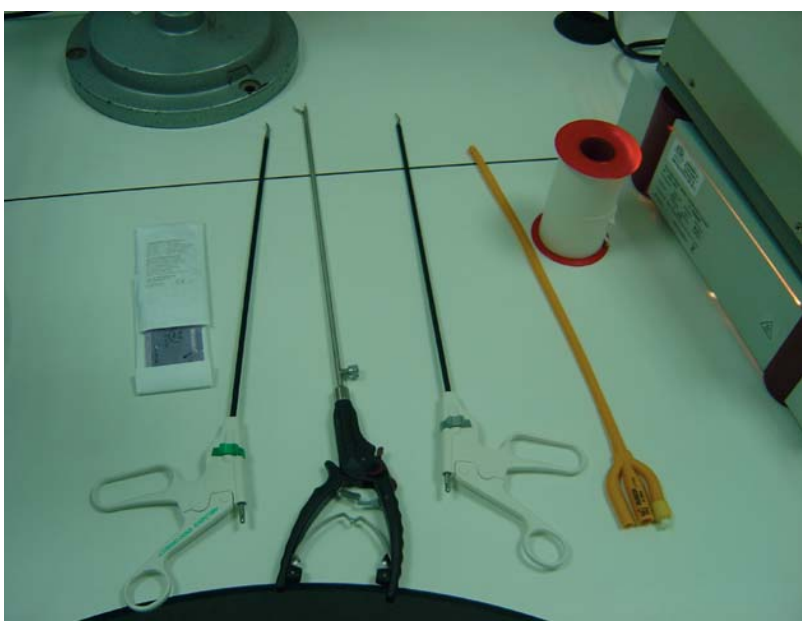

FIGURA 3: Ejemplo de material necesario para practicar en pelvitrainer.

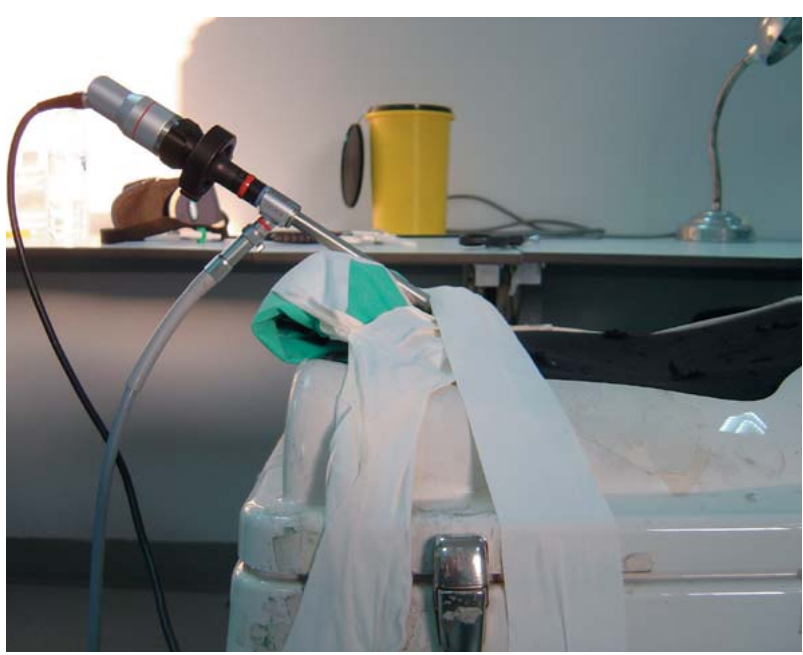

FIGURA 4: Detalle de la fijación de la cámara al pelvitrainer. 


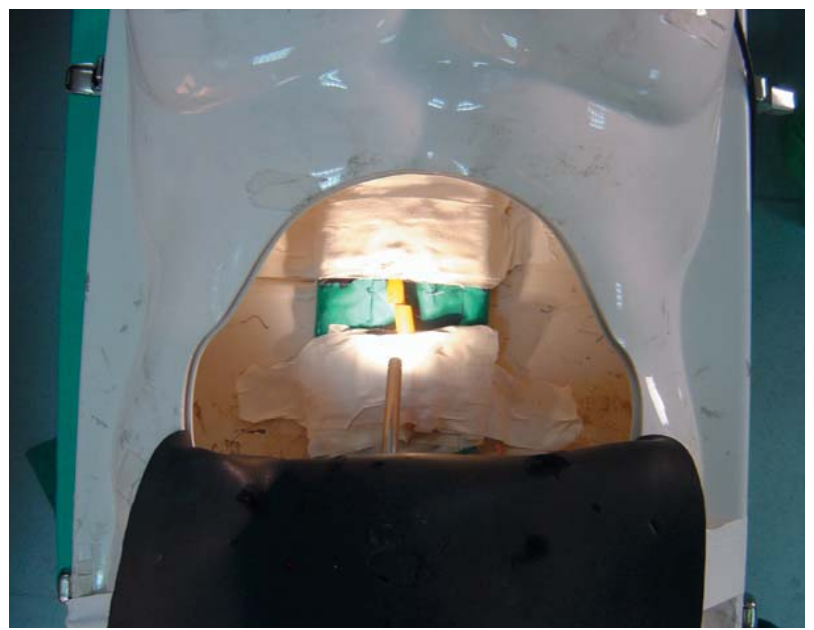

FIGURA 5: Modelo de anastomosis con sonda en pelvitrainer.

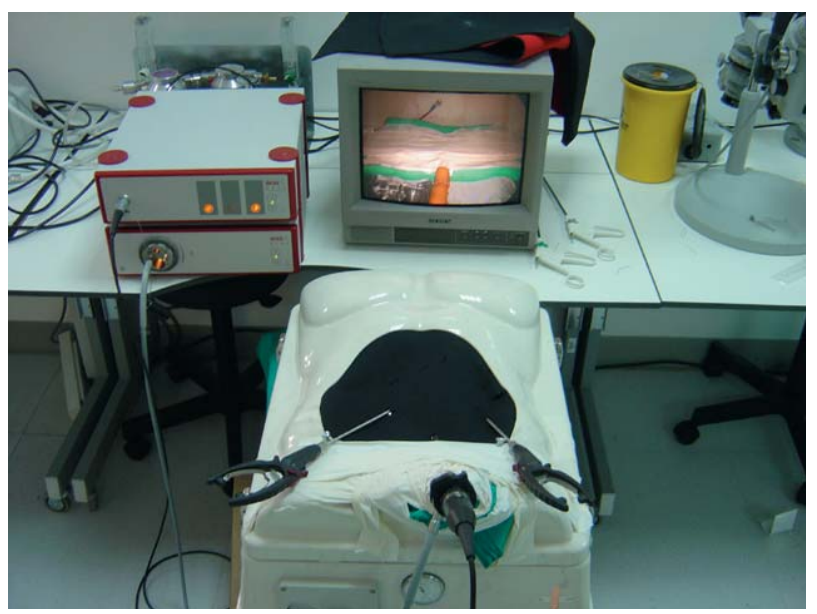

FIGURA 6: Pelvitrainer preparado para trabajar.

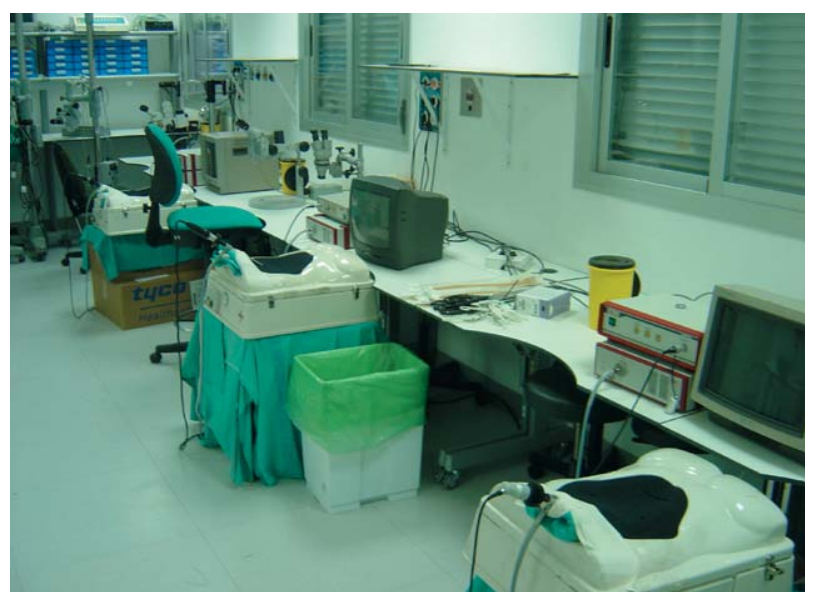

FIGURA 7: Practicaremos con porta y pinza o 2 portas.

el entrenamiento en otra tanda de animales de experimentación ( 3 cerdas) y se pasó a realizar la técnica en humanos.

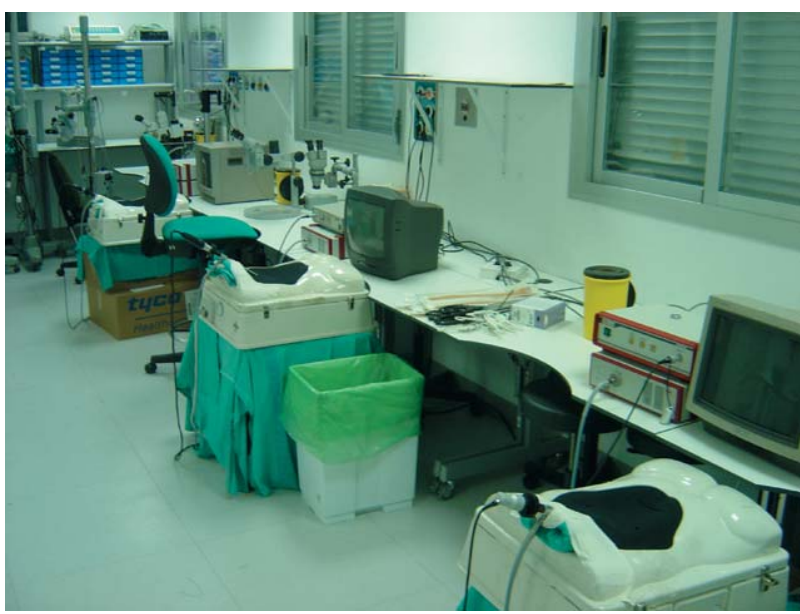

FIGURA 8: Sala de pelvitrainer del quirófano experimental H.U.La Paz.

Al finalizar la curva de aprendizaje de cada cirujano, comenzaron a ser ayudados por otros miembros del servicio y residentes, los cuales en la actualidad realizan la técnica como cirujanos, extendiendo así los conocimientos aprendidos y trasmitiendo su experiencia al resto del servicio.

Esto ha servido para dar solidez y consistencia al proyecto de laparoscopia y supone una garantía de futuro. Actualmente se han incorporado otros 3 miembros del servicio como primeros cirujanos así como los R-5 y se prevén más incorporaciones de forma progresiva.

Las personas que se van incorporando al programa de laparoscopia, al principio sólo llevan la cámara y el aspirador. Recordamos aquí, que nosotros realizamos la prostatectomía radical con un solo ayudante. Después de coger soltura con la cámara, comienzan a realizar ellos mismos los pasos más sencillos como descolgar vejiga, disección de vesículas seminales o sección de pedículos sin preservación de erectores, siempre tutorizados por un cirujano con experiencia. Finalmente, tras muchas ayudantías y muchas porciones de cirugias realizadas, completan su primera prostatectomía radical laparoscópica, no habiendo sido necesario el cambio de cirujano por imposibilidad de realizarla en ningún caso.

\section{Conclusiones}

El modelo para implantar con éxito la prostatectomía radical laparoscópica, en centros con una escasa experiencia previa incluye los siguientes pasos: 
1. Asegurarse de presentar una alta prevalencia de cánceres de próstata.

2. Elección de los urólogos que deben iniciar el programa.

3. Adiestramiento en simulador.

4. Experimentación en animales.

5. Rotación por centros con experiencia.

6. Aplicación de la técnica en humanos.

\section{CURVA DE APRENDIZAJE- COMPLICACIONES DE NUESTROS PRIMEROS 32 CASOS}

Analizamos las complicaciones ocurridas durante las primeras 32 cirugias en nuestro servicio.

En 4 casos fue necesario reconvertir a cirugía abierta.

Todos los casos se dieron dentro de las primeras 18 cirugías, lo que habla a favor de una estrecha relación con la poca experiencia en cirugía laparoscópica, puesto que en ningún otro caso posteriormente se ha precisado dicha medida.

De los 4 casos, el primero (caso 1) se debió a apertura vesical, mal control de la hemostasia y falta de experiencia.

El caso 6, a dificultad con la anastomosis.

El caso 12 presentó una hipercapnia y fibrilación auricular secundaria con imposibilidad de manejo anestésico. El paciente tenía una lobectomía pulmonar previa con una capacidad ventilatoria disminuida, lo cual junto con la falta de experiencia del equipo de anestesia condujo a acidosis respiratoria que ocasionó la fibrilación auricular.

En el caso 18, se provocó lesión iliaca e intestinal con la colocación de un trocar, debido a la fuerza necesaria para meter un trocar, por no haber hecho una apertura de piel suficiente.
Todos los casos, salvo el tercero por hipercapnia, se debieron a falta de experiencia. Posteriormente se han producido situaciones similares, con dificultad en la anastomosis, control de la hemostasia, lesión rectal... que hemos solucionado de forma laparoscópica.

Durante los primeros 32 casos, surgieron otras complicaciones intra o postoperatorias en otros 8 casos, en uno de los cuales fue necesario realizar una laparotomía por hemorragia postoperatoria. Las 4 reconversiones mencionadas anteriormente y 7 de las 8 complicaciones (2 hemorragias intensas, 2 aperturas de recto, 1 ileo paralítico, 1 cuadro de hematuria intensa y $1 \mathrm{de}$ fuga de orina por el drenaje) aparecieron en los primeros 18 casos. Desde el caso 19 al 32 sólo se produjo una complicación (apertura vesical) que se resolvió laparoscópicamente. El análisis de la gráfica de duración de la cirugía muestra una clara reducción a partir del $10^{\circ}$ caso, pasando de 469 minutos de media en las 10 primeras a 296 minutos en las siguientes y actualmente de 195 minutos en los últimos 100 casos. ( $\mathrm{p}<0,03$; $\mathrm{U}$ de Mann-Whitney) (Tabla 1).

Las gráficas de hemorragia, tiempo con drenaje y tiempo de estancia muestran también una clara reducción y disminución de la variabilidad, pero sólo a partir del caso 18 . A partir del $18^{\circ}$ caso la hemorragia media pasó de $1.250 \mathrm{cc}$ a $600 \mathrm{cc}$ y 200 cc en los últimos 100 casos. En cuanto al drenaje, pasó de una media de 3,1 días a 2,1 días y la estancia postoperatoria media de 5,1 días a 2,7 días en los últimos 100 casos $(\mathrm{p}<0,03$; $\mathrm{U}$ de Mann-Whitney).

\section{Conclusiones}

El análisis de nuestra curva de aprendizaje en prostatectomía radical laparoscópica muestra

Tabla 1

Descriptiva

\begin{tabular}{lcccccc}
\hline Casos & $\mathbf{1 - 5 0}$ & $\mathbf{5 1 - 1 0 0}$ & $\mathbf{1 0 1 - 1 4 7}$ & $\mathbf{1 4 8 - 2 0 0}$ & $\mathbf{2 0 1 - 2 6 2}$ & $\mathbf{2 6 2 - 5 4 4}$ \\
\hline Duración min & $300(135-630)$ & $211(105-300)$ & $210(110-330)$ & $195(110-390)$ & $181(90-420)$ & $197(105-270)$ \\
Hemorragia ml & $715(100-3500)$ & $374(100-120)$ & $389(100-1200)$ & $382(100-1200$ & $360(50-900)$ & $348(50-800)$ \\
Estancia días & $3,8(2-17)$ & $3(2-6)$ & $3(2-14)$ & $3,7(2-16)$ & $3,4(2-9)$ & $3,2(2-8)$ \\
Transfusión & $38 \%$ & $20 \%$ & $10,6 \%$ & 9,4 & $5,1 \%$ & $1 \%$ \\
\hline
\end{tabular}


que se requieren en torno a 20 procedimientos para llegar a una importante reducción en el número de complicaciones y reconversiones, así como en la hemorragia y en la estancia hospitalaria.

La revisión de la literatura en relación a la curva de aprendizaje en PRL, objetiva que, por norma general, el número de casos necesarios para vencer la tediosa curva de aprendizaje, es de aproximadamente $60-80 \operatorname{casos}^{1,2}$, a partir de los cuales, se comienza a disminuir de forma estable la duración de la cirugía, el sangrado y la tasa de complicaciones.

Quizás en nuestro caso, el hecho de tener a dos cirujanos aprendiendo al mismo tiempo, pudo favorecer el aprender uno de otro y disminuir así la curva de aprendizaje en el apartado de las complicaciones, no así en cuanto a duración, sangrado, conservación de cuello y haces neurovasculares, que sí requieren más casos para depurar la técnica.

Pensamos que el hecho de implicar a los residentes casi desde el principio, ha aportado solidez y garantía de futuro, puesto que garantiza el aprendizaje a dos generaciones al mismo tiempo.

Se dice que los residentes tienen mayor facilidad para aprender ésta técnica, pero pensamos que el secreto no se encuentra en que pertenezcamos a la generación de las nuevas tecnologías, sino a que se han dado los pasos correctos en el aprendizaje de cualquier técnica quirúrgica, iniciada por los miembros del servicio que creyeron en el futuro de la PRL e implantaron la técnica en nuestro servicio.

El residente ha estudiado la teoría, ha practicado con el pelvitrainer, y ha practicado con cerdos en el experimental. Otro aspecto importante es haber hecho cursos de laparoscopia en centros de reconocido prestigio. El paso final y fundamental, es haber participado como ayudante en un buen número de cirugías antes de pasar al puesto de cirujano. Esto le ha permitido adquirir experiencia a partir de los errores de sus antecesores, lo cual ha facilitado enormemente el aprendizaje y desarrollo de la técnica.

\section{REFERENCIAS}

1. Gregori A, Simonato A, Lissiani A, Bozzola A, Galli S, Gaboardi F. Laparoscopic radical prostatectomy: perioperative complications in an initial and consecutive series of 80 cases. Eur Urol. 2003;44(2):190-194.

2. Stolzenburg JU, Do M, Rabenalt R, Pfeiffer H, Horn L, Truss MC, Jonas U, Dorschner W. Endoscopic extraperitoneal radical prostatectomy: initial experience after 70 procedures. J Urol. 2003 Jun;169(6):2066-2071.

Dr. J.R. Cansino Alcaide

E-mail: urocansino@yahoo.es 